\title{
Associations between morphology and hemodynamics of intracranial aneurysms based on 4D flow and black-blood magnetic resonance imaging
}

\author{
Miaoqi Zhang ${ }^{1 \#}$, Fei Peng ${ }^{2,3,4 \#}$, Yunduo $\mathrm{Li}^{1}, \mathrm{Le} \mathrm{He}^{1}$, Aihua $\mathrm{Liu}^{2,3,4}$, Rui $\mathrm{Li}^{1}$ \\ ${ }^{1}$ Center for Biomedical Imaging Research, Department of Biomedical Engineering, School of Medicine, Tsinghua University, Beijing, China; ${ }^{2}$ Beijing \\ Neurosurgical Institute, Capital Medical University, Beijing, China; ${ }^{3}$ Department of Interventional Neuroradiology, Beijing Tiantan Hospital, \\ Capital Medical University, China; ${ }^{4}$ China National Clinical Research Center for Neurological Diseases, Beijing, China
}

\#These authors contributed equally to this work.

Correspondence to: Rui Li. Center for Biomedical Imaging Research, School of Medicine, Tsinghua University, Haidian District, Beijing 100084, China. Email: leerui@tsinghua.edu.cn; Aihua Liu. Beijing Neurosurgical Institute, Capital Medical University, Beijing 100050, China. Email: liuaihuadoctor@163.com.

Background: Previous studies have hypothesized that intracranial aneurysm (IA) morphology interacts with hemodynamic conditions. Magnetic resonance imaging (MRI) provides a single image modality solution for both morphological and hemodynamic measurements for IA. This study aimed to explore the interaction between the morphology and hemodynamics of IA using black-blood MRI (BB-MRI) and 4D flow MRI.

Methods: A total of 97 patients with unruptured IA were recruited for this study. The IA size, size ratio (SR), and minimum wall thickness (mWT) were measured using BB-MRI. Velocity, blood flow, pulsatility index (PI), and wall shear stress (WSS) were measured with 4D flow MRI. The relationship between hemodynamic parameters and morphological indices was investigated by linear regression analysis and unpaired two-sample $t$-test. To determine the independent interaction, multiple linear regression analysis was further performed.

Results: The findings showed that $\mathrm{mWT}$ was negatively correlated with IA size $(\mathrm{r}=-0.665, \mathrm{P}<0.001)$. Maximum blood flow in IA $\left(\mathrm{Flow}_{\mathrm{IA}}\right)$ was positively correlated with IA size $(\mathrm{r}=0.458, \mathrm{P}<0.001)$. The average WSS $\left(\mathrm{WSS}_{\text {avg }}\right)$ was negatively correlated with IA size $(\mathrm{r}=-0.650, \mathrm{P}<0.001)$. The relationships remained the same after the multivariate analysis was adjusted for hemodynamic, morphologic, and demographic confounding factors. The $\mathrm{WSS}_{\text {avg }}$ was positively correlated with $\mathrm{mWT}(\mathrm{r}=0.528, \mathrm{P}<0.001)$. In the unpaired two-sample $t$-test, $\mathrm{mWT}$, WSS $\mathrm{avg}_{\text {, }}$ and Flow $_{\mathrm{IA}}$ were statistically significantly associated with the size and SR of IAs.

Conclusions: There is potential for BB-MRI and 4D flow MRI to provide morphological and hemodynamic information regarding IA. Blood flow, WSS, and mW'T may serve as non-invasive biomarkers for IA assessments, and may contribute to a more comprehensive understanding of the mechanism of IA.

Keywords: Intracranial aneurysm (IA); black-blood magnetic resonance imaging (BB-MRI); 4D flow magnetic resonance imaging (4D flow MRI)

Submitted Mar 14, 2020. Accepted for publication Sep 18, 2020.

doi: 10.21037/qims-20-440

View this article at: http://dx.doi.org/10.21037/qims-20-440 


\section{Introduction}

Intracranial aneurysm (IA) refers to the local dilatation of a cerebral artery. IAs often occur in the circle of Willis, especially at arterial bifurcations (1). Rupture of IA, which is the most common cause of non-traumatic subarachnoid hemorrhage (SAH), carries a mortality rate of $50 \%$ (2). With the development of advanced imaging techniques, such as digital subtraction angiography (DSA), computed tomography angiography (CTA), and magnetic resonance angiography (MRA), IA can be detected before rupture occurs. Previous studies have suggested that morphological indices, including IA size (3) and IA size ratio (SR) (4), may serve as biomarkers of IA rupture. However, IA size and IA SR may not provide a comprehensive evaluation of IA rupture risk, because other characteristics, such as condition of the vessel walls (5) and hemodynamics (6), have been reported to play an important role in aneurysm rupture.

Previous studies have hypothesized that IA morphology interacts with hemodynamic conditions. The variation of morphology can change the hemodynamic conditions, including blood flow, velocity, pulsatility index (PI), and more advanced parameters, such as wall shear stress (WSS) (7). Moreover, hemodynamic conditions also affect IA size (8), IA wall thickness (9), size ratio (10), and other morphological parameters. However, most of these studies were performed on animal models or using computational fluid dynamics (CFD) simulation. Both the morphological and hemodynamic characteristics of IA need to be observed with advanced imaging techniques in a large group of IA patients. Recently, it has been reported that black-blood magnetic resonance imaging (BB-MRI) can be used to characterize IA size and wall thickness (WT) $(11,12)$. Moreover, the popularity of 4D flow MRI, which combines time-resolved $3 \mathrm{D}$ phase contrast MRI with postprocessing strategies, for the measurement of hemodynamic parameters in IA has increased (13-15). MRI provides a single image modality solution for both the morphological and hemodynamic measurements of IA.

Therefore, the purpose of this study was to analyze the interaction of morphology and hemodynamics in a large group of IA patients using BB-MRI and 4D flow MRI techniques.

\section{Methods}

\section{Study population}

This study involved 97 patients $[26$ male and 71 females; age, $57.7 \pm 11.6$ years (range, $20-81$ years)] with unruptured IA detected by DSA, CTA, or MRA who were recruited between April 2014 and October 2018. Patients with saccular IAs $>4 \mathrm{~mm}$ were included. Patients with contraindications to MRI, including pregnancy or breastfeeding, claustrophobia, or existing MRI-incompatible implants were excluded, as were patients whose MRI scans were incomplete or had poor image quality.

\section{Standard protocol approvals, registrations, and patient consents}

All protocols were reviewed and approved by the local institutional ethics committee, and written informed consent was obtained from all participants.

\section{In vivo MR imaging}

All MR imaging was performed using a 3.0T MR scanner (Achieva TX, Philips, Best, The Netherlands) with a 32-channel head coil. First, a 3D black-blood T1-weighted volumetric isotropic turbo spin echo acquisition (T1VISTA) sequence (12) was used to investigate IA size and wall conditions. The imaging parameters were TR/ TE $=800 / 21 \mathrm{~ms}, \mathrm{FOV}=200 \times 180 \times 40 \mathrm{~mm}^{3}$, voxel size $=0.6 \times 0.6 \times 0.6 \mathrm{~mm}^{3}$, echo train length $(\mathrm{ETL})=30,2$ startup echoes, and number of averages $=1$. The variable flip angle scheme was set according to a previously reported study (16). Further, 4D flow MRI data was acquired by a freebreathing, peripheral pulse-gated, multi-shot turbo field echo sequence for hemodynamic parameters evaluation. The scan parameters were TR/TE $=8.0 / 3.6 \mathrm{~ms}$, FOV $=160 \times 160 \times 30 \mathrm{~mm}^{3}$, voxel size $=1 \times 1 \times 1 \mathrm{~mm}^{3}$, velocity encoding $(V E N C)=120 \mathrm{~cm} / \mathrm{s}$ for all 3 directions, flip angle $=20^{\circ}$, turbo factor $=3,4$ startup echoes, and number of averages $=1$. Parallel imaging was applied to accelerate the acquisition for T1-VISTA and 4D flow, with a sensitivity encoding (SENSE) factor of 2 and 3 in a right-to-left direction, respectively.

\section{Morphological analysis}

The IA size measured by BB-MRI was defined as the largest value of: (I) aneurysm neck width, which was the virtual line separating the aneurysm from the parental artery; (II) maximum aneurysm height, which was the maximum height from the neck to the dome tip; or (III) maximum aneurysm width, which was perpendicular to maximum aneurysm 


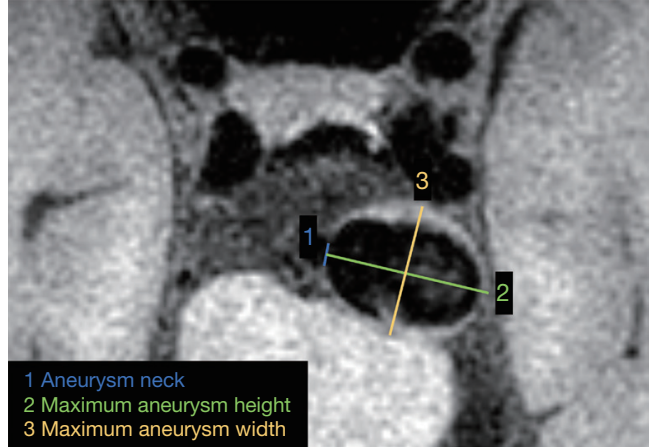

Figure 1 Measurement of IA size using BB-MRI. IA size was defined as the largest of three measurements. IA, intracranial aneurysm; BB-MRI, black-blood magnetic resonance imaging.

height, as illustrated in Figure 1.

The IA SR was calculated by dividing the maximum aneurysm height by the mean parent artery diameter on BB-MRI $(10,17)$. The minimum wall thickness (mWT) of the IA was measured in the same geometry as BB-MRI as WSS measurements (in hemodynamic analysis), which will be introduced in the next section. If the $\mathrm{mWT}$ of the IA > voxel size, the wall thickness was directly measured manually. For aneurysm walls that were thinner than voxel sizes, wall thickness was calculated using the following equation (18):

$$
w=d \frac{S_{w}}{S_{0}}
$$

where $S_{w}$ is the voxel signal, $d$ is the voxel size, $S_{0}$ is the voxel signal completely filled with vessel wall.

\section{Hemodynamic analysis}

All preprocessing, visualization, and quantification of 4D flow data were performed using GTFlow, version 2.2.15 (GyroTools LLC., Zurich, Switzerland). Preprocessing steps, including eddy current correction, velocity mask application, and vessel segmentation, were performed prior to data visualization and quantification. Velocity-weighted masks were adopted to segment the IA, and streamlines were provided for visualization and quantification of blood flow.

Hemodynamic parameters including velocity, blood flow, PI, and WSS were measured at the peak systole, (when peak velocity appears) on 4D flow MRI scan. A cross-sectional plane was created perpendicular to the streamline containing the maximum velocity point with IA (Figure 2) and contours were drawn manually. By evaluating all pixels within the contours, maximum through-plane velocity $\left(\mathrm{V}_{\mathrm{IA}}, \mathrm{cm} / \mathrm{s}\right)$ and blood flow through the cut-plane (Flow ${ }_{\text {IA }}, \mathrm{mL} / \mathrm{s}$ ) were measured, and PI was calculated as follows:

$$
P I=\frac{V_{\text {peak }}-V_{\text {valley }}}{V_{\text {mean }}}
$$

where $V_{\text {peak }} / V_{\text {valley }} / V_{\text {mean }}$ are peak/valley/mean values of maximum through-plane velocity $(\mathrm{cm} / \mathrm{s})$ over all time indices in 1 cardiac cycle in the same cross-sectional plane as defined previously. Furthermore, WSS was defined as the velocity gradient along the perpendicular path of the IA wall $(19,20)$. Average WSS (WSS ${ }_{\text {avg, }} \mathrm{N} / \mathrm{m}^{2}$ ) magnitude along the contour was calculated. The hemodynamic parameters in the adjacent parent artery (APA) of the aneurysms, including maximum through-plane velocity $\left(\mathrm{V}_{\mathrm{APA}}, \mathrm{cm} / \mathrm{s}\right)$, and maximum blood flow (Flow ${ }_{\mathrm{APA}}, \mathrm{mL} / \mathrm{s}$ ), were also explored at the cross-sectional plane where maximum velocity appeared (Figure 2) (21). All definitions of hemodynamic parameter measurements are summarized and illustrated in Figure 3.

\section{Statistical analysis}

Statistical analysis was conducted with MedCalc (version 15.2, MedCalc Software bvba, Mariakerke, Belgium) and MATLAB (version 8.5.0 (R2017a), MathWorks Inc., Natick, MA, USA). The relationship between IA size and other factors including the morphological indices of $\mathrm{mWT}$, hemodynamic parameters $V_{\mathrm{IA}}, V_{\mathrm{APA}}$, Flow $\mathrm{IA}_{\mathrm{IA}}$, Flow $_{\mathrm{APA}}$, PI, and $\mathrm{WSS}_{\text {avg, }}$, and the demographic parameters of sex, age, smoking history, hypertension, diabetes, and hyperlipidemia was investigated by linear regression analysis $(r)$. The variables which $\mathrm{P}<0.2$ were selected for multivariate logistic regression analysis (partial correlation $r$ ). The relationship between $\mathrm{WSS}_{\text {avg }}$ and $\mathrm{mWT}$ was also investigated by linear regression analysis $(r)$. Furthermore, the unpaired twosample $t$-test was used to investigate the association between IA size, IA SR, and hemodynamic parameters. The level of statistical significance was set at $\mathrm{P}<0.05$.

\section{Results}

Of the 97 patients examined in the study period, 27 participants were excluded due to incomplete MRI scans or poor image quality. Therefore, 70 patients (mean age, 

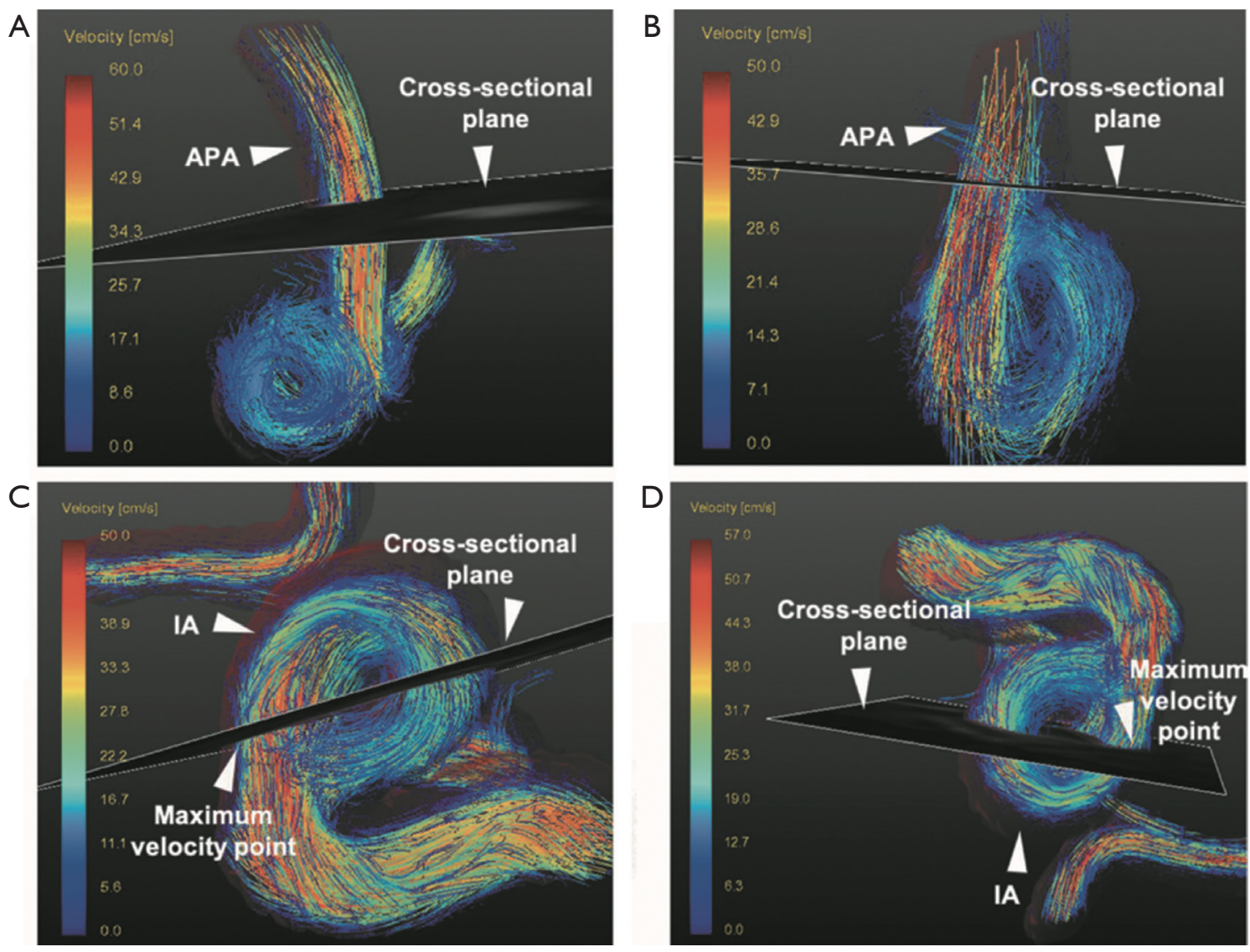

Figure 2 Illustration of 4D flow data analysis using dedicated software in different IA. Flow pattern visualization of the (A,B) APA and (C,D) IA were performed by streamlines, and hemodynamic measurements within contours were conducted in a defined cross-sectional plane of the APA and IA. APA, adjacent parent artery; IA, intracranial aneurysm.

$53.8 \pm 11.7$ years) with 72 aneurysms were included.

The relationship between morphological and hemodynamic parameters with potentially demographic confounding factors such as sex, age, smoking history, hypertension, diabetes, and hyperlipidemia are summarized in Table 1. A negative correlation was found between mWT and IA size $(\mathrm{r}=-0.665, \mathrm{P}<0.001)$ and $\mathrm{WSS}_{\text {avg }}$ and IA size $(\mathrm{r}=-0.650, \mathrm{P}<0.001)$. A positive correlation existed between Flow $_{\text {IA }}$ and IA size $(r=0.458, \mathrm{P}<0.001)$. The relationship still existed when the multivariate analysis was adjusted for confounding factors. The $\mathrm{WSS}_{\text {avg }}$ were positively correlated with $\mathrm{mWT}(\mathrm{r}=0.528, \mathrm{P}<0.001)$. Furthermore, scatter plots and regression lines were used to demonstrate those three relationships (Figure 4).

The unpaired two-sample $t$-test was used to further analyze the association between morphological and hemodynamic parameters. The relationships between IA diameter (abscissa) and 7 features (ordinate) are summarized in Figure 5A. The relationships between IA SR (abscissa) and 7 features (ordinate) are summarized in Figure 5B. The participants were divided into 2 subgroups based on the features. For 7 non-binary features (PI, Flow ${ }_{\mathrm{IA}}, \mathrm{V}_{\mathrm{IA}}$, Flow $\mathrm{APA}_{\mathrm{AP}}$, $\mathrm{V}_{\mathrm{APA}}, \mathrm{WSS}_{\text {age, }}$ and $\mathrm{mWT}$ ), the median was chosen as the threshold. As shown in Figure 5 A, Flow $_{\mathrm{IA}}$, WSS ${ }_{\text {avg, }}$ and $\mathrm{mWT}$ were statistically significantly associated with the IA size. These results were consistent with the linear regression in Figure 4, in which $\mathrm{mWT}$, as well as $\mathrm{WSS}_{\text {avg }}$, were negatively correlated with IA size, whereas Flow IA $_{\text {IA }}$ was positively related to the IA size. Figure $5 B$ shows that $\mathrm{Flow}_{\mathrm{IA}}, \mathrm{WSS}_{\text {avg, }}$ and $\mathrm{mWT}$ were statistically significantly associated with IA SR.

Figure 6 shows two examples of patients with small (Figure 6A,B) and large (Figure 6C,D) IAs, respectively. The large IA had a thinner mWT, smaller WSS, and faster maximum blood flow than the small IA. 


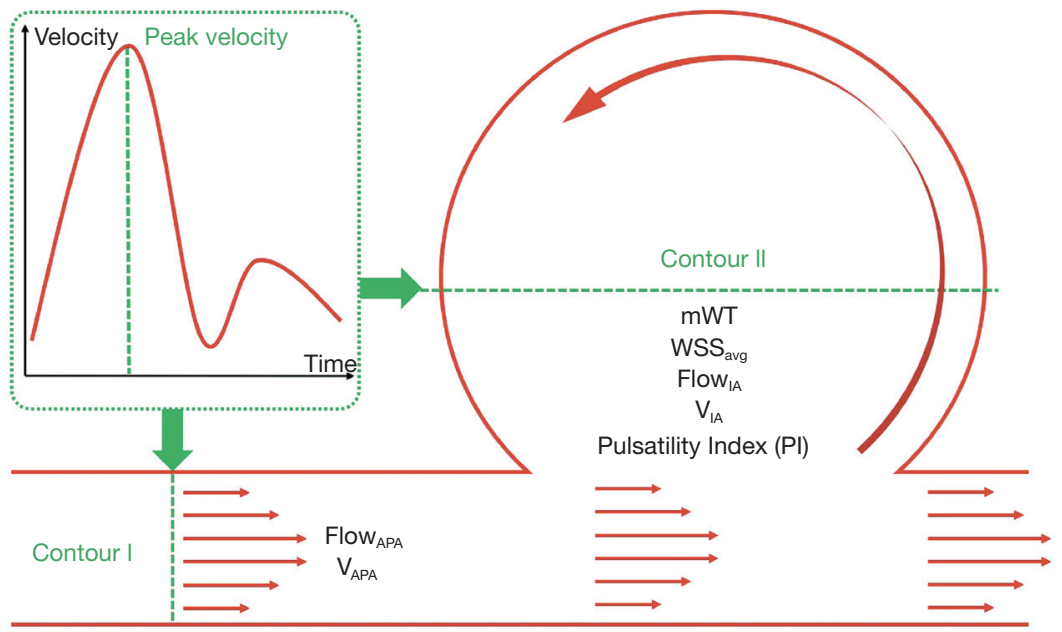

Figure 3 Illustration of hemodynamic and morphological parameter measurements. All hemodynamic measurements were implemented at the peak systolic phase. Cut-planes were created in the largest cross-sectional plane of APA and IA containing the maximum velocity vector. Maximum through-plane velocity in the APA $\left(\mathrm{V}_{\mathrm{APA}}, \mathrm{cm} / \mathrm{s}\right)$ and maximum blood flow in the APA (Flow $\mathrm{APA}_{\mathrm{AP}}$, $\left.\mathrm{s}\right)$ were automatically measured. Maximum through-plane velocity in the IA $\left(\mathrm{V}_{\mathrm{IA}}, \mathrm{cm} / \mathrm{s}\right)$, maximum blood flow in the IA (Flow $\mathrm{IA}_{\mathrm{I}}$, $\left.\mathrm{mL} / \mathrm{s}\right)$, and average WSS of the IA $\left(\mathrm{WSS}_{\text {avg }}\right.$ ) were also automatically measured. In the meantime, mWT was also measured in the same geometry from BB-MRI. APA, adjacent parent artery; IA, intracranial aneurysm; mWT, minimum wall thickness; WSS, wall shear stress.

Table 1 The relationship between IA size and other factors including morphological index

\begin{tabular}{|c|c|c|c|c|}
\hline \multirow[t]{2}{*}{ Factors } & \multicolumn{2}{|c|}{ Univariate analysis } & \multicolumn{2}{|c|}{$\begin{array}{l}\text { Multivariate analysis } \\
\left(R^{2}=0.647, P<0.0001\right)\end{array}$} \\
\hline & r & $\mathrm{P}$ & rpartial & $P$ \\
\hline $\mathrm{mWT}(\mathrm{mm})$ & -0.665 & $<0.001^{*}$ & -0.334 & $0.006^{*}$ \\
\hline FlowiA (mL/s) & 0.458 & $<0.001^{\star}$ & 0.276 & $0.025^{\star}$ \\
\hline FlowAPA (mL/s) & 0.217 & 0.067 & 0.157 & 0.207 \\
\hline $\mathrm{V}_{\mathrm{IA}}(\mathrm{cm} / \mathrm{s})$ & 0.101 & 0.400 & - & - \\
\hline$V_{A P A}(\mathrm{~cm} / \mathrm{s})$ & 0.037 & 0.756 & - & - \\
\hline$W_{S S}$ avg $\left(\mathrm{N} / \mathrm{m}^{2}\right)$ & -0.650 & $<0.001^{*}$ & -0.522 & $<0.001^{*}$ \\
\hline $\mathrm{PI}$ & -0.112 & 0.350 & - & - \\
\hline Sex & 0.042 & 0.727 & - & - \\
\hline Age & -0.230 & 0.053 & -0.166 & 0.184 \\
\hline Smoking history & -0.070 & 0.559 & - & - \\
\hline Hypertension & -0.078 & 0.513 & - & - \\
\hline Diabetes & -0.183 & 0.124 & 0.080 & 0.524 \\
\hline Hyperlipidemia & -0.222 & 0.062 & -0.008 & 0.950 \\
\hline
\end{tabular}

mWT hemodynamic parameters: ViA, VAPA, FloWIA, FloWAPA, PI, and WSSavg, and demographic parameters: sex, age, smoking history, hypertension, diabetes, and hyperlipidemia. * $\mathrm{P}<0.05$. IA, intracranial aneurysm; VIA, velocity of IA; VAPA, velocity of adjacent parent artery; WSSavg, average wall shear stress; PI, pulsatility index.

\section{Discussion}

In this study, BB-MRI and 4D flow MRI were applied to acquire multiple imaging biomarkers of IAs, and the relationships between morphological and hemodynamic parameters were analyzed.

First, we found that blood flow in IAs was positively correlated with size, even after multivariate analysis was adjusted for confounding factors. Previous studies have reported that hemodynamics play an important role in the pathogenesis and treatment of IA $(22,23)$. Some of these studies found that the formation of aneurysms relied on an increase in blood flow (24), hinting that an increase in blood flow may also be required to increase the IA size. Also, by leading to an increment of IA pressure, increased blood flow might contribute to growth in the size of the IA; a recent study suggested that aneurysm pressure was positively correlated with aneurysm size (25). Since IA size has been considered as a predictor of IA rupture, it is reasonable to state that hemodynamics, especially for large blood flow, may also be taken as an important parameter for the risk evaluation of IA rupture. Second, we found that $\mathrm{WSS}_{\text {avg }}$ was negatively correlated with IA size. Our findings support the results of a previous study (7) in which the WSS showed a negative linear correlation with the aneurysm dome size, suggesting that the WSS may decrease 
A

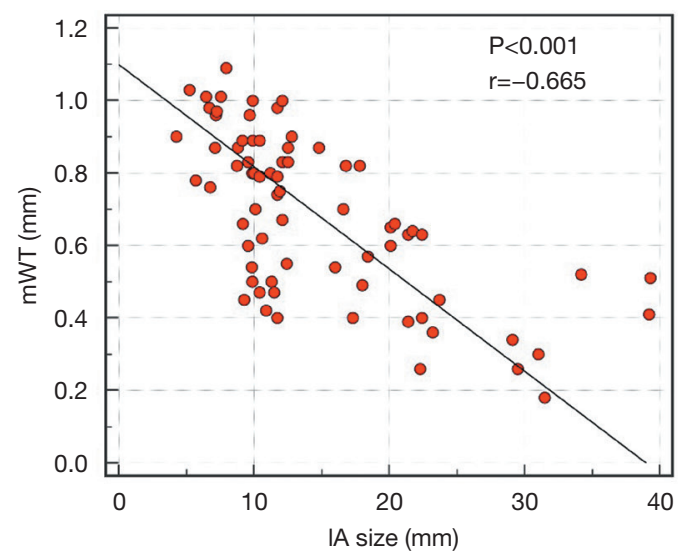

C

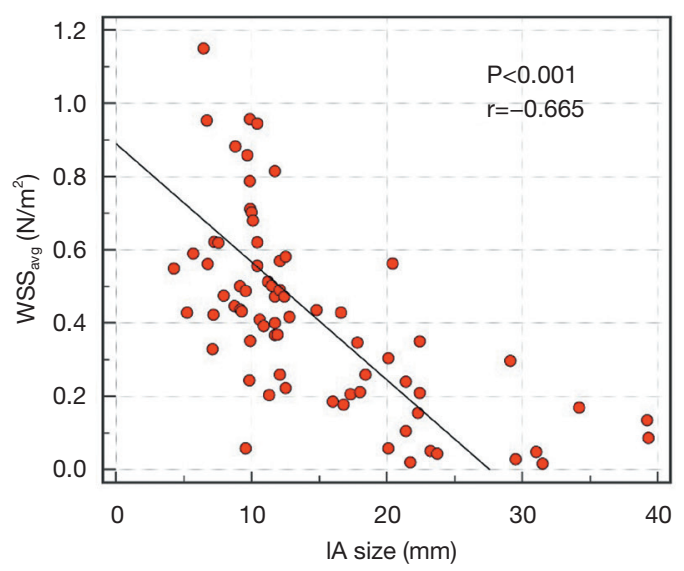

B

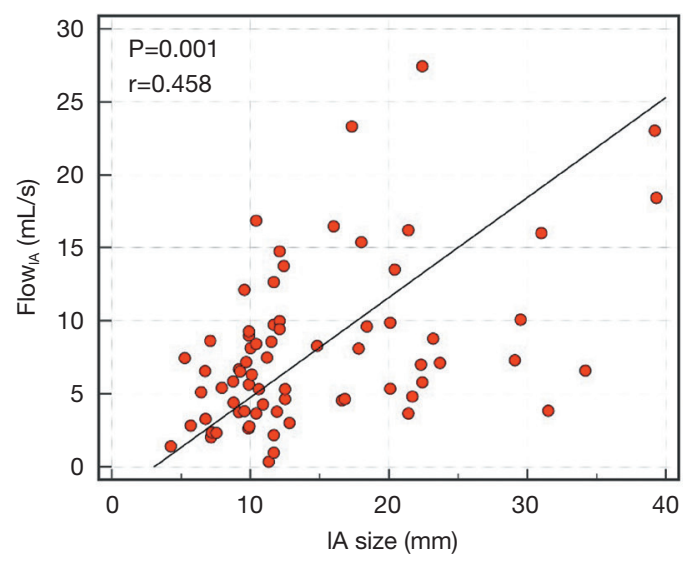

D

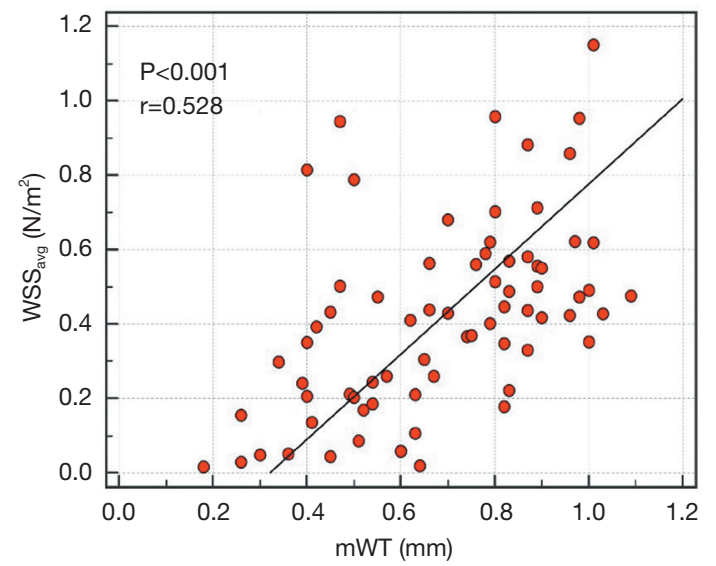

Figure 4 Scatter plots illustrating the relationship between morphological indices and hemodynamic parameters. (A) mWT was negatively

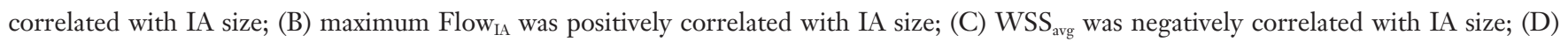
WSS $_{\text {avg }}$ was positively correlated with mWT. mWT, minimum wall thickness; WSS, wall shear stress.

as the IA grows. Third, we found that $\mathrm{WSS}_{\text {avg }}$ was positively correlated with $\mathrm{mWT}$. It is still unclear whether high or low WSS is associated with aneurysm wall degeneration (26-29) or IA growth/rupture (30). Our findings support that low WSS may be associated with the process of aneurysm wall degeneration. This finding is similar to that of a previous study (9), which reported that thin-walled regions of unruptured cerebral aneurysms colocalize with low WSS. Researchers have also theorized that low WSS may contribute to wall degradation through a static deleterious endothelial phenotype $(31,32)$, which has been indirectly illustrated by our results.

A similar relationship to that between IA size and hemodynamics was found in the relationship between IA SR and hemodynamics (Figure 5), with SR representing the ratio between IA size and parent artery diameter. These results indicated that the parent artery diameter had a minimal effect on the hemodynamic condition of aneurysms. This is consistent with the fact that Flow ${ }_{\mathrm{APA}}$ and $\mathrm{V}_{\mathrm{APA}}$ are not associated with IA size (Table 1, Figure 5). Although Kashiwazaki et al. (17) showed that IA SR was a predictive factor for rupture, their criteria included patients with small IAs (IAs $<5 \mathrm{~mm}$ ), whereas our analysis included patients with IAs $>4 \mathrm{~mm}$. This difference in entry criteria may be the primary reason for the different conclusions.

Interestingly, we also found that blood flow velocity was not associated with IA size. Considering that blood flow is the integral of velocity with respect to contour area, and contour area is determined by IA size, it is reasonable to state that $\mathrm{V}_{\text {IA }}$ may not be a risk factor for aneurysm rupture. 
A

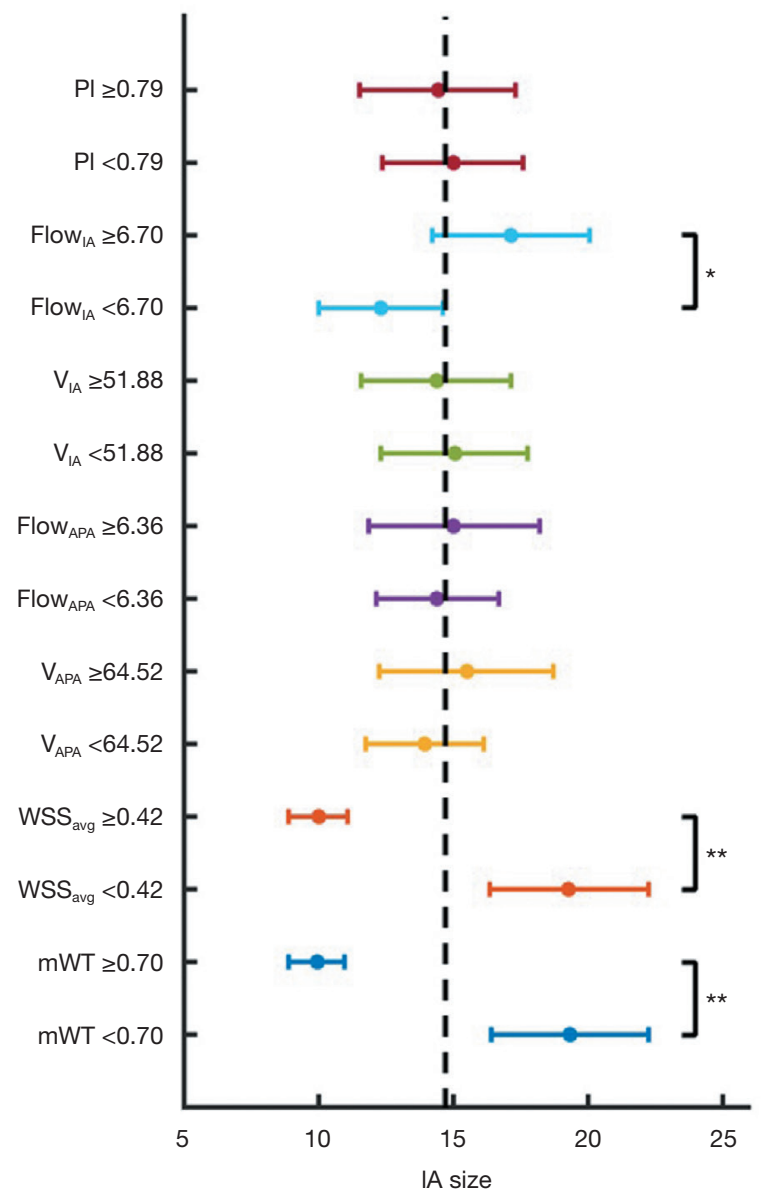

B

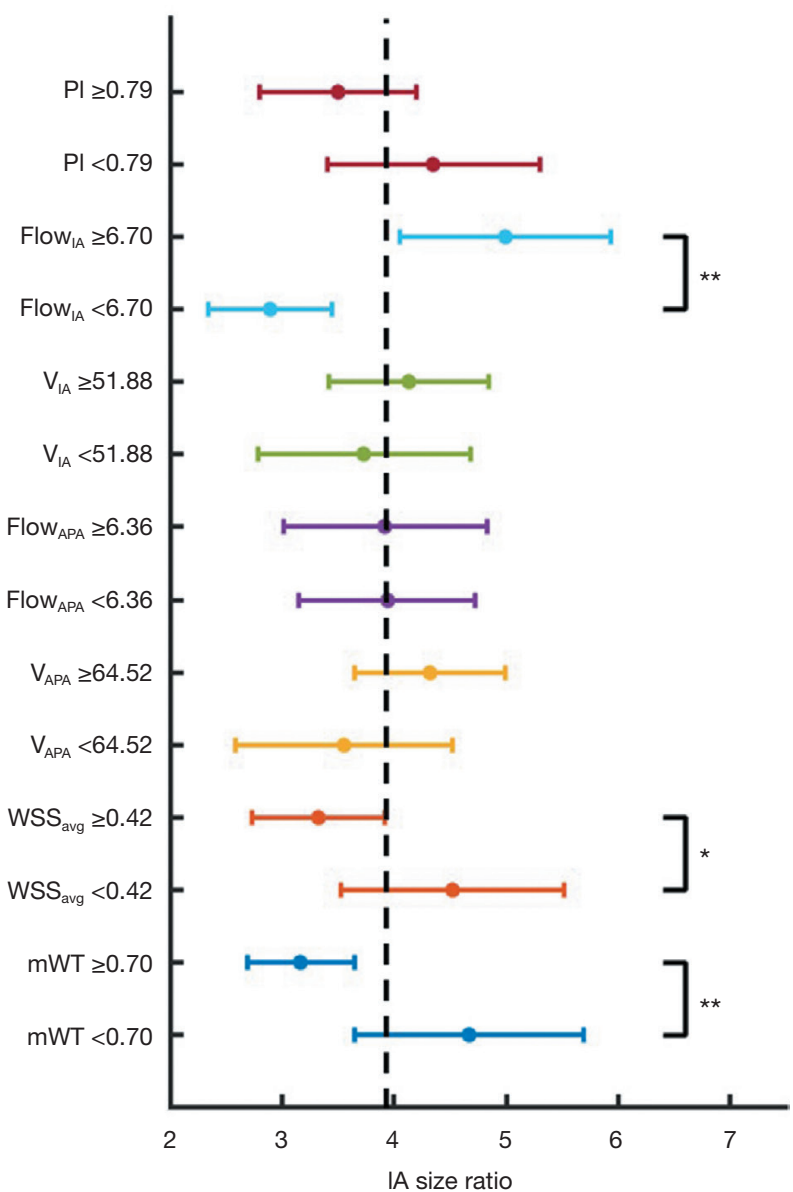

Figure 5 (A) The relationship (A) between IA diameter (abscissa) and 7 features (ordinate) and (B) between IA size ratio (abscissa) and 7 features (ordinate). For each feature, patients were separated into two subgroups based on the feature. Mean IA diameter, mean IA size ratio (SR), and 95\% CI are shown for all subgroups. Vertical dashed lines show the mean IA diameter and mean IA SR from 72 patients. Two sample $t$-test. *, $\mathrm{P}<0.05$; ${ }^{* *}, \mathrm{P}<0.01$. IA, intracranial aneurysm.

Additionally, PI was not associated with morphological indices, which demonstrated that PI may not be a risk factor for aneurysm rupture.

Currently, the most widely used IA rupture risk evaluation method in clinical practice is the aneurysm sizebased standard, as established by the International Study of Unruptured Intracranial Aneurysms (ISUIA) (33). One major drawback of the aneurysm size-based ISUIA standard is that some small aneurysms may still rupture. Orz et al. (34) investigated 34 patients who had small, as well as medium or large-sized aneurysm(s); of the 34 participants, 9 (26.5\%) displayed rupture of the small aneurysms. Their data suggest that small aneurysms ( $\leq 6 \mathrm{~mm}$ in size, ISUIA grade 1 ) are prone to rupture. In this study, larger aneurysms were observed to have increased blood flow, smaller WSS, and smaller mWT. However, some small aneurysms (ISUIA grade 1 , size $\leq 7 \mathrm{~mm}$ ) were found to have a faster blood flow, smaller WSS, and smaller mWT. These results suggest that other than size, blood flow, WSS, and mWT may be monitored to provide additional information about aneurysm instability.

A benefit of the high resolution of BB-MRI is that it can be used to provide detailed morphological information of IAs (18). By measuring IA size and mWT simultaneously, BB-MRI has demonstrated these two parameters to be negatively correlated. Moreover, 4D flow MRI can provide a more versatile depiction of IA hemodynamics than other imaging techniques, and its accuracy and stability 

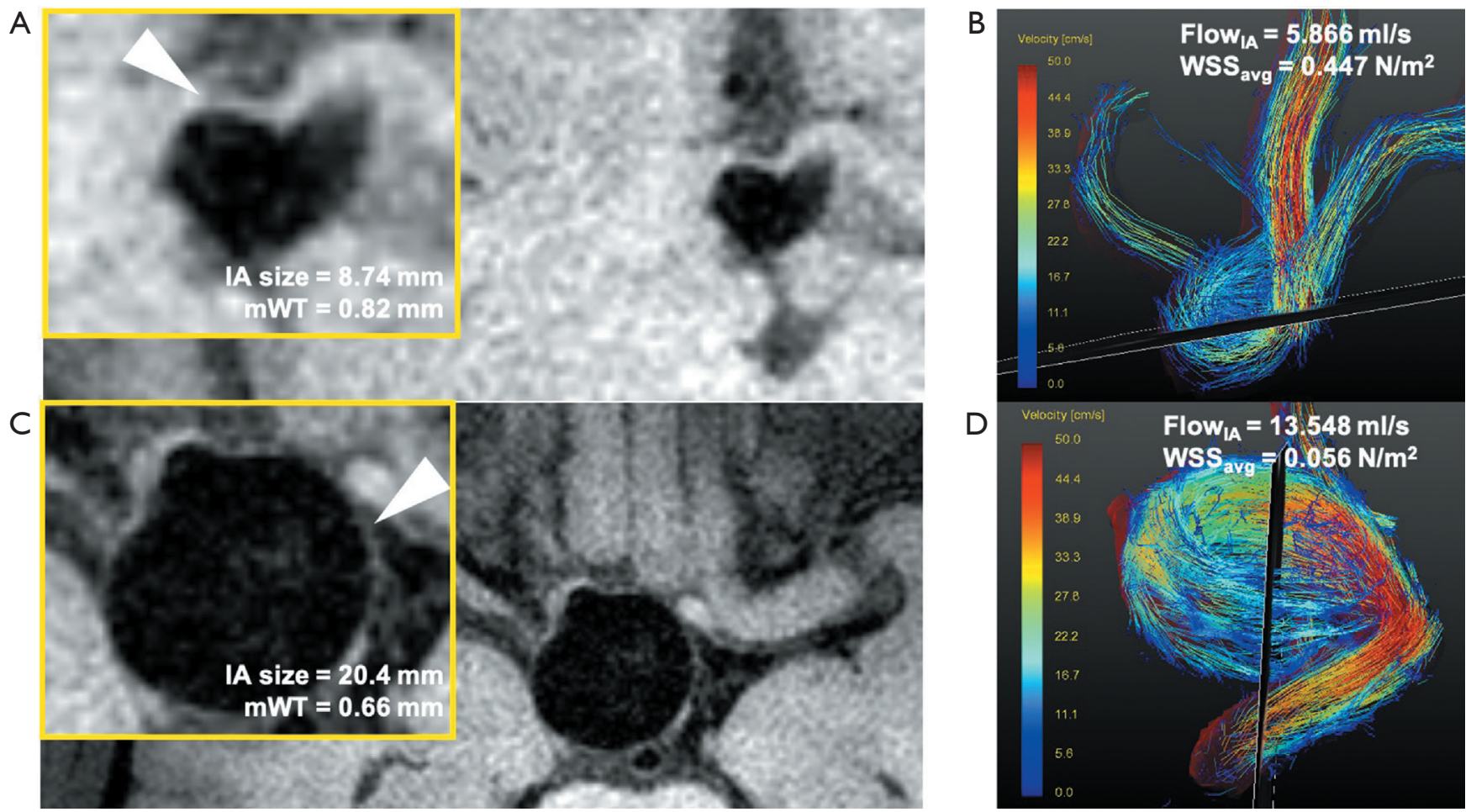

Figure 6 Two representative cases of IA. Example of a patient with: $(\mathrm{A}, \mathrm{B}) \mathrm{IA}$ size $=8.74 \mathrm{~mm}, \mathrm{mWT}=0.82 \mathrm{~mm}$, $\mathrm{maximum} \mathrm{Flow}_{\mathrm{IA}}=5.866 \mathrm{~mL} /$ $\mathrm{s}, \mathrm{WSS}_{\text {avg }}=0.447 \mathrm{~N} / \mathrm{m}^{2}$, and another patient with (C,D) IA size $=20.4 \mathrm{~mm}, \mathrm{mWT}=0.66 \mathrm{~mm}, \mathrm{Flow}_{\mathrm{IA}}=13.548 \mathrm{~mL} / \mathrm{s}, \mathrm{WSS}_{\text {avg }}=0.056 \mathrm{~N} / \mathrm{m}^{2}$. The giant IA has a thinner wall thickness, smaller WSS, and faster maximum blood flow than the small IA. IA, intracranial aneurysm; mWT, minimum wall thickness; WSS, wall shear stress.

on hemodynamic measurements have been acknowledged $(10,35,36)$. Another alternative to 4D Flow MRI is using hemodynamic software based on CFD simulations, such as the analysis system (ANSYS). The CFD software calculates hemodynamic parameters based on flow model, boundary conditions, and simulation. It has several disadvantages, including: (I) simulation modeling is hypothetical and uncertain (37); (II) low sensitivity of reconstructed geometry to imaging modality (38); (III) segmentation variability (39); and (VI) unrealistic modeling of flow data (10). On the contrary, 4D flow MRI and related post-processing software provide velocity in three directions at each voxel during a cardiac cycle measured with the principle of phase contrast MRI measures velocity using MRI physics, and is not restricted by model precision, boundary conditions, or fluid structure interaction (FSI). By combining BBMRI and 4D flow MRI, the relationship between the morphology and hemodynamics of IA can be further illustrated. More importantly, BB-MRI and 4D flow MRI may help to characterize the aneurysm wall and abnormal hemodynamics overlooked by conventional imaging modalities, which may further guide treatment selection.

This study has some limitations. First, the measurement of morphological parameters (mWT, IA size, and IA SR) was conducted manually, which increases the chance of error. Also, due to the limited spatial resolution of 4D flow MRI, patients with IA sizes $<4 \mathrm{~mm}$ were not included; our conclusions may not be generalizable on small aneurysms. Moreover, longitudinal studies are required to investigate the relationship between imaging biomarkers and rupture risk of IA.

\section{Conclusions}

In summary, by utilizing BB-MRI and 4D flow MRI, four associations between morphology and hemodynamics of IA were found. First, mWT was negatively correlated with IA size; second, blood flow in IA was positively correlated with IA size; third, WSS was negatively correlated with IA size; and fourth, WSS was positively correlated with $\mathrm{mWT}$. 
Blood flow, WSS, and $\mathrm{mWT}$ measured by 4D flow and BBMRI may be used as non-invasive biomarkers to assess IAs, and contribute to a more comprehensive characterization of the IA mechanism.

\section{Acknowledgments}

The authors thank Qingchu Jin and Shuo Chen, Ph.D, for their assistance and advice in undertaking this study.

Funding: This work was supported by the Natural Science Foundation of China (81971604, R Li), the Natural Science Foundation of China (81771233, A Liu), and the Natural Science Foundation of Beijing (L192013, R Li \& A Liu).

\section{Footnote}

Conflicts of Interest: All authors have completed the ICMJE uniform disclosure form (available at http://dx.doi. org/10.21037/qims-20-440). The authors have no conflicts of interest to declare.

Ethical Statement: This study was approved by the Institutional Review Board at Tsinghua University, and all the subjects provided written informed consent.

Open Access Statement: This is an Open Access article distributed in accordance with the Creative Commons Attribution-NonCommercial-NoDerivs 4.0 International License (CC BY-NC-ND 4.0), which permits the noncommercial replication and distribution of the article with the strict proviso that no changes or edits are made and the original work is properly cited (including links to both the formal publication through the relevant DOI and the license). See: https://creativecommons.org/licenses/by-nc-nd/4.0/.

\section{References}

1. Bonneville F, Sourour N, Biondi A. Intracranial aneurysms: an overview. Neuroimaging Clin N Am 2006;16:371-82.

2. van Gijn J, Kerr RS, Rinkel GJ. Subarachnoid haemorrhage. Lancet 2007;369:306-18.

3. Sluzewski M, Bosch JA, van Rooij WJ, Nijssen PC, Wijnalda D. Rupture of intracranial aneurysms during treatment with Guglielmi detachable coils: incidence, outcome, and risk factors. J Neurosurg 2001;94:238-40.

4. Rahman M, Smietana J, Hauck E, Hoh B, Hopkins N, Siddiqui A, Levy EI, Meng H, Mocco J. Size ratio correlates with intracranial aneurysm rupture status: a prospective study. Stroke 2010;41:916-20.

5. Steiger HJ, Aaslid R, Keller S, Reulen HJ. Strength, elasticity and viscoelastic properties of cerebral aneurysms. Heart Vessels 1989;5:41-6.

6. Schnell S, Ansari SA, Vakil P, Wasielewski M, Carr ML, Hurley MC, Bendok BR, Batjer H, Carroll TJ, Carr J, Markl M. Three-dimensional hemodynamics in intracranial aneurysms: influence of size and morphology. J Magn Reson Imaging 2014;39:120-31.

7. Tateshima S, Chien A, Sayre J, Cebral J, Viñuela F. The effect of aneurysm geometry on the intra-aneurysmal flow condition. Neuroradiology 2010;52:1135-41.

8. Gao L, Hoi Y, Swartz DD, Kolega J, Siddiqui A, Meng H. Nascent aneurysm formation at the basilar terminus induced by hemodynamics. Stroke 2008;39:2085-90.

9. Kadasi LM, Dent WC, Malek AM. Colocalization of thinwalled dome regions with low hemodynamic wall shear stress in unruptured cerebral aneurysms. J Neurosurg 2013;119:172-9.

10. Futami K, Nambu I, Kitabayashi T, Sano H, Misaki K, Uchiyama N, Nakada M. Inflow hemodynamics evaluated by using four-dimensional flow magnetic resonance imaging and the size ratio of unruptured cerebral aneurysms. Neuroradiology 2017;59:411-8.

11. Sakurai K, Miura T, Sagisaka T, Hattori M, Matsukawa N, Mase M, Kasai H, Arai N, Kawai T, Shimohira M, Yamawaki T, Shibamoto Y. Evaluation of luminal and vessel wall abnormalities in subacute and other stages of intracranial vertebrobasilar artery dissections using the volume isotropic turbo-spin-echo acquisition (VISTA) sequence: A preliminary study. J Neuroradiol 2013;40:19-28.

12. Liu P, Qi H, Liu A, Lv X, Jiang Y, Zhao X, Li R, Lu B, Lv M, Chen H, Li Y. Relationship between aneurysm wall enhancement and conventional risk factors in patients with unruptured intracranial aneurysms: A black-blood MRI study. Interv Neuroradiol 2016;22:501-5.

13. Boussel L, Rayz V, Martin A, Acevedo-Bolton G, Lawton MT, Higashida R, Smith WS, Young WL, Saloner D. Phase-contrast magnetic resonance imaging measurements in intracranial aneurysms in vivo of flow patterns, velocity fields, and wall shear stress: comparison with computational fluid dynamics. Magn Reson Med 2009;61:409-17.

14. Wetzel S, Meckel S, Frydrychowicz A, Bonati L, Radue EW, Scheffler K, Hennig J, Markl M. In vivo assessment and visualization of intracranial arterial hemodynamics with flow-sensitized 4D MR imaging at 3T. AJNR Am J 
Neuroradiol 2007;28:433-8.

15. Hope TA, Hope MD, Purcell DD, von Morze C, Vigneron DB, Alley MT, Dillon WP. Evaluation of intracranial stenoses and aneurysms with accelerated 4D flow. Magn Reson Imaging 2010;28:41-6.

16. Qiao Y, Steinman DA, Qin Q, Etesami M, Schär M, Astor BC, Wasserman BA. Intracranial arterial wall imaging using three-dimensional high isotropic resolution black blood MRI at 3.0 Tesla. J Magn Reson Imaging 2011;34:22-30.

17. Kashiwazaki D, Kuroda S; Sapporo SAH Study Group. Size ratio can highly predict rupture risk in intracranial small (<5 mm) aneurysms. Stroke 2013;44:2169-73.

18. Blankena R, Kleinloog R, Verweij BH, van Ooij P, Ten Haken B, Luijten PR, Rinkel GJ, Zwanenburg JJ. Thinner Regions of Intracranial Aneurysm Wall Correlate with Regions of Higher Wall Shear Stress: A 7T MRI Study. AJNR Am J Neuroradiol 2016;37:1310-7.

19. Stalder AF, Russe MF, Frydrychowicz A, Bock J, Hennig J, Markl M. Quantitative 2D and 3D phase contrast MRI: optimized analysis of blood flow and vessel wall parameters. Magn Reson Med 2008;60:1218-31.

20. Harloff A, Albrecht F, Spreer J, Stalder AF, Bock J, Frydrychowicz A, Schöllhorn J, Hetzel A, Schumacher M, Hennig J, Markl M. 3D blood flow characteristics in the carotid artery bifurcation assessed by flow-sensitive 4D MRI at 3T. Magn Reson Med 2009;61:65-74.

21. Li Y, Chen H, He L, Cao X, Wang X, Chen S, Li R, Yuan C. Hemodynamic assessments of venous pulsatile tinnitus using 4D-flow MRI. Neurology 2018;91:e586-93.

22. Jou LD, Quick CM, Young WL, Lawton MT, Higashida R, Martin A, Saloner D. Computational approach to quantifying hemodynamic forces in giant cerebral aneurysms. AJNR Am J Neuroradiol 2003;24:1804-10.

23. Cebral JR, Castro MA, Appanaboyina S, Putman CM, Millan D, Frangi AF. Efficient pipeline for imagebased patient-specific analysis of cerebral aneurysm hemodynamics: technique and sensitivity. IEEE Trans Med Imaging 2005;24:457-67.

24. Song J, Park JE, Kim HR, Shin YS. Observation of cerebral aneurysm wall thickness using intraoperative microscopy: clinical and morphological analysis of translucent aneurysm. Neurol Sci 2015;36:907-12.

25. Doenitz C, Schebesch KM, Zoephel R, Brawanski A. A mechanism for the rapid development of intracranial aneurysms: a case study. Neurosurgery 2010;67:1213-21.

26. Chien A, Tateshima S, Castro M, Sayre J, Cebral J, Viñuela F. Patient-specific flow analysis of brain aneurysms at a single location: comparison of hemodynamic characteristics in small aneurysms. Med Biol Eng Comput 2008;46:1113-20.

27. Shojima M, Oshima M, Takagi K, Torii R, Hayakawa M, Katada K, Morita A, Kirino T. Magnitude and role of wall shear stress on cerebral aneurysm: computational fluid dynamic study of 20 middle cerebral artery aneurysms. Stroke 2004;35:2500-5.

28. Boussel L, Rayz V, McCulloch C, Martin A, AcevedoBolton G, Lawton M, Higashida R, Smith WS, Young WL, Saloner D. Aneurysm growth occurs at region of low wall shear stress: patient-specific correlation of hemodynamics and growth in a longitudinal study. Stroke 2008;39:2997-3002.

29. Tateshima S, Tanishita K, Omura H, Villablanca JP, Vinuela F. Intra-aneurysmal hemodynamics during the growth of an unruptured aneurysm: in vitro study using longitudinal CT angiogram database. AJNR Am J Neuroradiol 2007;28:622-7.

30. Meng H, Tutino VM, Xiang J, Siddiqui A. High WSS or low WSS? Complex interactions of hemodynamics with intracranial aneurysm initiation, growth, and rupture: toward a unifying hypothesis. AJNR Am J Neuroradiol 2014;35:1254-62.

31. Dai G, Kaazempur-Mofrad MR, Natarajan S, Zhang Y, Vaughn S, Blackman BR, Kamm RD, García-Cardeña G, Gimbrone MA Jr. Distinct endothelial phenotypes evoked by arterial waveforms derived from atherosclerosissusceptible and -resistant regions of human vasculature. Proc Natl Acad Sci U S A 2004;101:14871-6.

32. Malek AM, Alper SL, Izumo S. Hemodynamic shear stress and its role in atherosclerosis. JAMA 1999;282:2035-42.

33. Wiebers DO, Whisnant JP, Huston J 3rd, Meissner I, Brown RD Jr, Piepgras DG, Forbes GS, Thielen K, Nichols D, O'Fallon WM, Peacock J, Jaeger L, Kassell NF, Kongable-Beckman GL, Torner JC; International Study of Unruptured Intracranial Aneurysms Investigators. Unruptured intracranial aneurysms: natural history, clinical outcome, and risks of surgical and endovascular treatment. Lancet 2003;362:103-10.

34. Orz Y, Kobayashi S, Osawa M, Tanaka Y. Aneurysm size: a prognostic factor for rupture. Br J Neurosurg 1997;11:144-9.

35. Dyverfeldt P, Bissell M, Barker AJ, Bolger AF, Carlhäll CJ, Ebbers T, Francios CJ, Frydrychowicz A, Geiger J, Giese D, Hope MD, Kilner PJ, Kozerke S, Myerson S, Neubauer S, Wieben O, Markl M. 4D flow cardiovascular magnetic resonance consensus statement. J Cardiovasc 
Magn Reson 2015;17:72.

36. Liu J, Koskas L, Faraji F, Kao E, Wang Y, Haraldsson H, Kefayati S, Zhu C, Ahn S, Laub G, Saloner D. Highly accelerated intracranial 4D flow MRI: evaluation of healthy volunteers and patients with intracranial aneurysms. MAGMA 2018;31:295-307.

37. Khan MO, Steinman DA, Valen-Sendstad K. NonNewtonian versus numerical rheology: Practical impact of shear-thinning on the prediction of stable and unstable flows in intracranial aneurysms. Int J Numer Method Biomed Eng 2017. doi: 10.1002/cnm.2836.

38. Geers AJ, Larrabide I, Radaelli AG, Bogunovic H, Kim M, Gratama van Andel HA, Majoie CB, VanBavel E, Frangi AF. Patient-specific computational hemodynamics of intracranial aneurysms from 3D rotational angiography and CT angiography: an in vivo reproducibility study.

Cite this article as: Zhang M, Peng F, Li Y, He L, Liu A, Li R. Associations between morphology and hemodynamics of intracranial aneurysms based on 4D flow and black-blood magnetic resonance imaging. Quant Imaging Med Surg 2021;11(2):597-607. doi: 10.21037/qims-20-440
AJNR Am J Neuroradiol 2011;32:581-6.

39. Berg P, Voß S, Saalfeld S, Janiga G, Bergersen AW, Valen-Sendstad K, Bruening J, Goubergrits L, Spuler A, Cancelliere NM, Steinman DA, Pereira VM, Chiu TL, Tsang ACO, Chung BJ, Cebral JR, Cito S, Pallarès J, Copelli G, Csippa B, Paál G, Fujimura S, Takao H, Hodis S, Hille G, Karmonik C, Elias S, Kellermann K, Khan MO, Marsden AL, Morales HG, Piskin S, Finol EA, Pravdivtseva M, Rajabzadeh-Oghaz H, Paliwal N, Meng H, Seshadhri S, Howard M, Shojima M, Sugiyama SI, Niizuma K, Sindeev S, Frolov S, Wagner T, Brawanski A, Qian Y, Wu YA, Carlson KD, Dragomir-Daescu D, Beuing O. Multiple Aneurysms AnaTomy CHallenge 2018 (MATCH): Phase I: Segmentation. Cardiovasc Eng Technol 2018;9:565-81. 